\title{
Dislocation related droop in InGaN/GaN light emitting diodes investigated via cathodoluminescence
}

Galia Pozina, Rafal Ciechonski, Zhaoxia Bi, Lars Samuelson and Bo Monemar

\author{
Linköping University Post Print
}

\section{Tweet}

N.B.: When citing this work, cite the original article.

Original Publication:

Galia Pozina, Rafal Ciechonski, Zhaoxia Bi, Lars Samuelson and Bo Monemar, Dislocation related droop in InGaN/GaN light emitting diodes investigated via cathodoluminescence, 2015, Applied Physics Letters, (107), 25, 251106.

http://dx.doi.org/10.1063/1.4938208

Copyright: American Institute of Physics (AIP)

http://www.aip.org/

Postprint available at: Linköping University Electronic Press

http://urn.kb.se/resolve?urn=urn:nbn:se:liu:diva-125162 


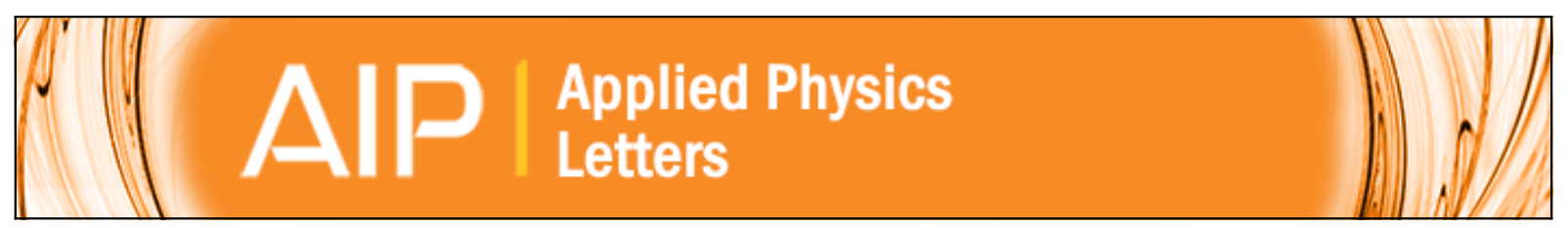

\section{Dislocation related droop in InGaN/GaN light emitting diodes investigated via cathodoluminescence}

Galia Pozina, Rafal Ciechonski, Zhaoxia Bi, Lars Samuelson, and Bo Monemar

Citation: Applied Physics Letters 107, 251106 (2015); doi: 10.1063/1.4938208

View online: http://dx.doi.org/10.1063/1.4938208

View Table of Contents: http://scitation.aip.org/content/aip/journal/apl/107/25?ver=pdfcov

Published by the AIP Publishing

\section{Articles you may be interested in}

High-power low-droop violet semipolar $\left(303^{-} 1^{-}\right)$InGaN/GaN light-emitting diodes with thick active layer design

Appl. Phys. Lett. 105, 171106 (2014); 10.1063/1.4900793

Influence of dislocation density on carrier injection in InGaN/GaN light-emitting diodes operated with alternating current

Appl. Phys. Lett. 102, 011115 (2013); 10.1063/1.4773588

Dependence of radiative efficiency and deep level defect incorporation on threading dislocation density for InGaN/GaN light emitting diodes

Appl. Phys. Lett. 101, 162102 (2012); 10.1063/1.4759003

Improvements of external quantum efficiency of InGaN-based blue light-emitting diodes at high current density using GaN substrates

J. Appl. Phys. 101, 033104 (2007); 10.1063/1.2432307

Effects of In composition on ultraviolet emission efficiency in quaternary InAIGaN light-emitting diodes on freestanding $\mathrm{GaN}$ substrates and sapphire substrates

J. Appl. Phys. 98, 113514 (2005); 10.1063/1.2134885

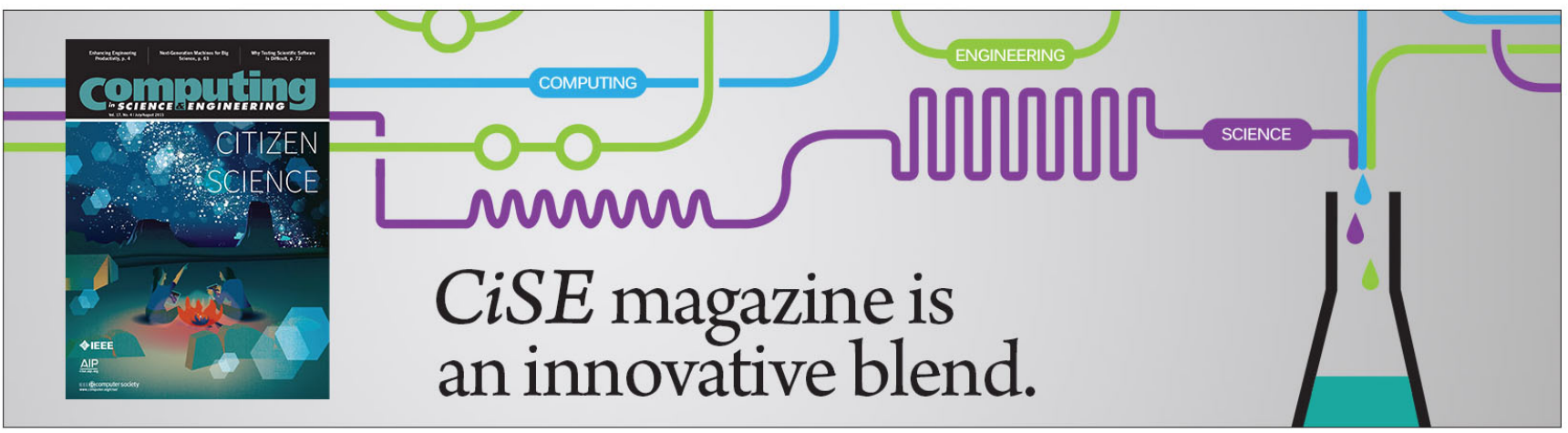




\title{
Dislocation related droop in InGaN/GaN light emitting diodes investigated via cathodoluminescence
}

\author{
Galia Pozina, ${ }^{1}$ Rafal Ciechonski, ${ }^{2}$ Zhaoxia Bi, ${ }^{3}$ Lars Samuelson,,${ }^{2,3}$ and Bo Monemar ${ }^{1,3,4}$ \\ ${ }^{1}$ Department of Physics, Chemistry and Biology, Linköping University, SE-581 83 Linköping, Sweden \\ ${ }^{2}$ GLO AB, Scheelevägen 22, SE-22363 Lund, Sweden \\ ${ }^{3}$ Solid State Physics, Lund University, Box 118, SE-22100 Lund, Sweden \\ ${ }^{4}$ TokyoUniversity of Agriculture and Technology, Koganei, Tokyo 184-8588, Japan
}

(Received 10 November 2015; accepted 7 December 2015; published online 23 December 2015)

\begin{abstract}
Today's energy saving solutions for general illumination rely on efficient white light emitting diodes (LEDs). However, the output efficiency droop experienced in InGaN based LEDs with increasing current injection is a serious limitation factor for future development of bright white LEDs. We show using cathodoluminescence (CL) spatial mapping at different electron beam currents that threading dislocations are active as nonradiative recombination centers only at high injection conditions. At low current, the dislocations are inactive in carrier recombination due to local potentials, but these potentials are screened by carriers at higher injection levels. In CL images, this corresponds to the increase of the dark contrast around dislocations with the injection (excitation) density and can be linked with droop related to the threading dislocations. Our data indicate that reduction of droop in the future efficient white LED can be achieved via a drastic reduction of the dislocation density by using, for example, bulk native substrates. (C) 2015 AIP Publishing LLC. [http://dx.doi.org/10.1063/1.4938208]
\end{abstract}

The reduction of radiative efficiency in InGaN based light emitting diodes (LEDs) for visible light with increasing current injection, the so-called droop, is a definite limitation for future improvement of the light output from these LEDs, including the related white lamps. ${ }^{1}$ The physical mechanisms for the droop have been discussed intensely during the last decade, as reviewed by several authors. ${ }^{1-3}$ These models are typically based on the assumption of homogeneous material properties in the active region producing the light, i.e., defects are not discussed as a major reason for droop. Some previous work pointed out that defect recombination could be viewed as consistent with the droop behavior in the LEDs, however. ${ }^{4,5}$ At injection conditions close to the peak of the LED efficiency curve (like external quantum efficiency vs current density, often called the LI-curve), the injected carriers are generally viewed as largely localized and, thus, immune to the major non-radiative defect recombination at dislocations. At high injection the localization potentials are screened, and the carriers are free to move to defect sites. ${ }^{4}$ Other authors have shown using high resolution scanning near-field optical microscopy techniques, which the dislocations themselves are protected from nonradiative recombination events by local potentials for the violet InGaN LEDs, under the conditions of these experiments. ${ }^{6}$ This property leaves them inactive in carrier recombination, unless these potentials are screened by carriers at higher injection levels. ${ }^{4}$ In this scenario, dislocations may play an important role for droop, because in these violet (or blue) LEDs they are essentially active as nonradiative recombination centers only at high injection conditions.

The dominating growth technologies for the nitride LEDs at present employ foreign substrates like sapphire, $\mathrm{SiC}$ or silicon, leading to a high threading dislocation (TD) density of $\sim 10^{9} \mathrm{~cm}^{-2}$ in the active region of the device, unless specific actions to reduce the dislocation density are taken. In this letter, we study an InGaN-based LED structure grown heteroepitaxially on a sapphire substrate and, thus, it is a suitable object to investigate the role of dislocations for droop. These dislocations are generally known to be important nonradiative centers at room temperature and above (as relevant for LEDs). ${ }^{7-9}$ At present, there is no published investigation of the spatial dependence of droop across an LED wafer, in order to demonstrate whether the droop is a homogeneous intrinsic effect (as presently assumed in most theoretical models). The intrinsic recombination processes are expected to be evenly distributed at high excitation conditions (free carrier recombination), and produce a homogeneous bright background in a cathodoluminescence (CL) topograph of the emission from a certain area of the LED wafer. If defects like TDs are involved an inhomogeneous emission pattern across the LED wafer is expected, with dark spots at the defect sites. ${ }^{7-9}$ If this dark contrast increases with the injection (excitation) density, we are observing droop related to the TDs.

The LED structure investigated in this work is a conventional violet $\mathrm{GaN} / \mathrm{InGaN}$ multiple quantum well (MQW) structure grown with metalorganic chemical vapor deposition at Lund University on a patterned sapphire substrate, including a 5 quantum well active region, an $\mathrm{AlGaN}$ electron blocking layer, and a $p$-layer. The contact metallization was excluded. On-wafer test with the Ni-dot method confirmed satisfactory emission performance. Room temperature CL measurements were performed using a MonoCL4 system integrated with a field emission gun (FEG) cathode LEO 1550 Gemini scanning electron microscope (SEM). The acceleration voltage of the electron beam was kept to $10 \mathrm{kV}$. With this voltage, the penetration depth is $\sim 1 \mu \mathrm{m}$ in the case of GaN material. The threading dislocation density was 

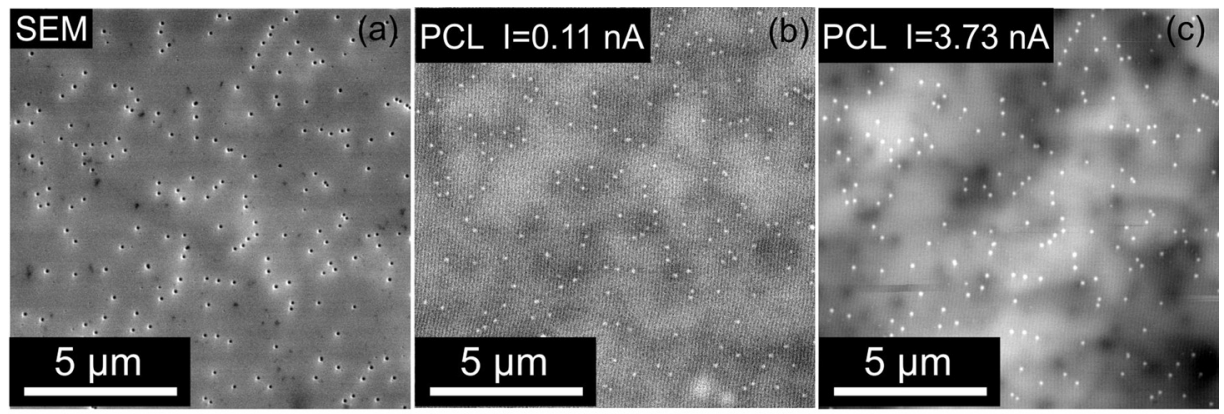

SEM

(d)

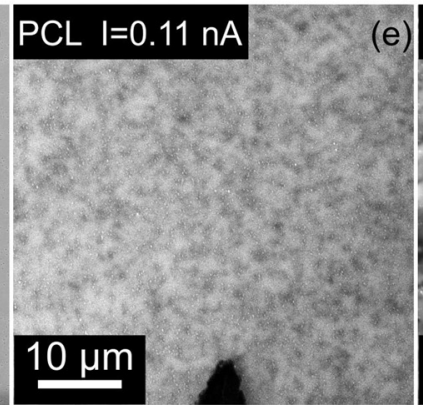

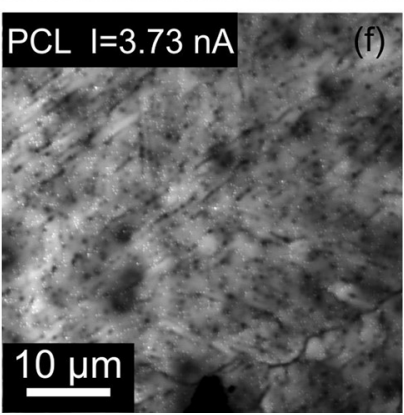

FIG. 1. SEM surface pictures (a) and (d) and corresponding panchromatic CL images taken from the same area of the wafer at two different electron beam currents (b) and (e) $0.11 \mathrm{nA}$ and (c) and (f) $3.73 \mathrm{nA}$. The images in (d)-(f) are similar to those in (a)-(c), but shown with lower magnification to underline a pronounced CL contrast around dislocations of different geometry at higher electron beam current. estimated from the dark spot density of $\sim 5 \times 10^{7} \mathrm{~cm}^{-2}$. The SEM instrument allows six different apertures, which were used to vary the intensity of the electron beam. Additionally, the beam current was measured for each aperture separately. $\mathrm{CL}$ images were acquired using a Peltier-cooled GaAs photomultiplier tube. A diffraction grating with $150 \mathrm{l} / \mathrm{mm}$ blazed at $500 \mathrm{~nm}$ and a CCD detector were used for the CL spectral measurements. The spectral resolution under the experimental conditions was $\sim 5 \mathrm{~nm}$. The spatial resolution of the CL system was better than $100 \mathrm{~nm}$, which is similar to the SEM resolution for the conditions of the experiment.

Panchromatic CL (PCL) images measured at room temperature for low and high electron beam current show different distribution of the CL signal, which can be described as a transformation from more uniform CL intensity to a clearly pronounced contrast decorating dislocations as dark spots and lines at the high intensity. We exemplify such behavior of the CL signal in Fig. 1, where PCL maps taken under different magnification and excitation intensities of the electron beam current are shown along with SEM images. The effect of the electron beam current on CL intensity distribution is obvious, especially for the data shown in Fig. 1(f) for lower magnification, when the dislocation network over a larger area can be clearly seen.

The situation looks similar at higher magnification as shown in Fig. 2. Here individual dark spots originating from TDs are observed, and the contrast around these spots clearly increases with the injection current. (The small spots present in these figures are related to near surface defects in the p- layer, caused by somewhat excessive p-doping. These spots appear dark in the SEM pictures but bright in the CL. These spots have no relation to the dislocation contrast from the active region CL.)

We have attempted to follow the evolution of the integrated intensity of the MQW emission with increasing electron beam current, for a small local area of the wafer. As noted from the CL images, the signal is not uniform. The bright contrast, i.e., a higher signal, corresponds to the areas with less structural defects (dislocations), while darker contrast can be related to the areas with the higher dislocation density. As already mentioned, the contrast in CL images is especially obvious for a higher electron current beam. We have selected two points with low and high CL signal, labeled as P1 and P2 in Fig. 3, respectively. The electron beam was focused at each point under spectra acquisition, while the beam current was varied. Although a small adjustment of focus, position and astigmatism was necessary to do, the points of measurements could be carefully followed for different apertures. Examples of CL spectra taken for the "bright" point P1 and for the "dark" point P2 are shown in Figs. 4(a) and 4(b) for the highest electron beam current of $4.19 \mathrm{nA}$ and for a relatively low current of $0.12 \mathrm{nA}$, respectively. Since the electron beam was focused to the spot with a diameter $<50 \mathrm{~nm}$, the injection current density is estimated to $\sim 6$ and $\sim 210 \mathrm{~A} / \mathrm{cm}^{2}$, respectively, comparable with operation currents of an LED. An integrated intensity of the MQW emission line versus the electron beam current is plotted in Fig. 4(c) for points P1 and P2.
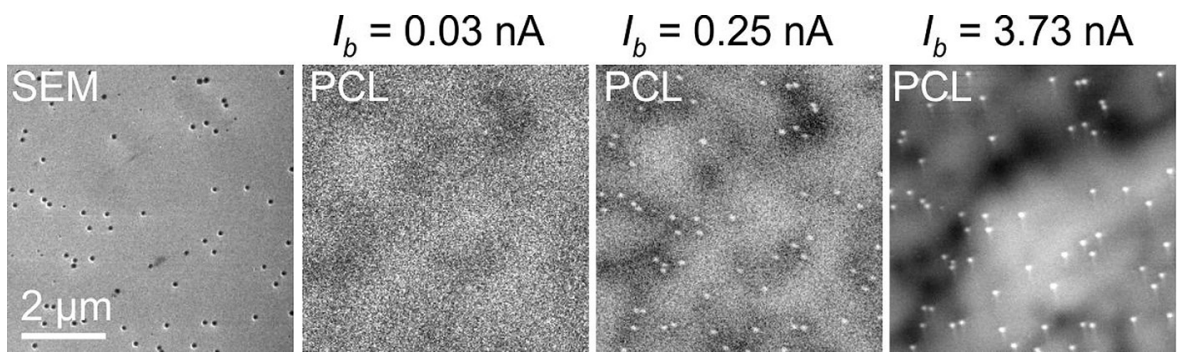

FIG. 2. Higher resolution picture of PCL contrast of individual dislocations versus excitation current. Also shown is a related SEM image with a scale marker. 


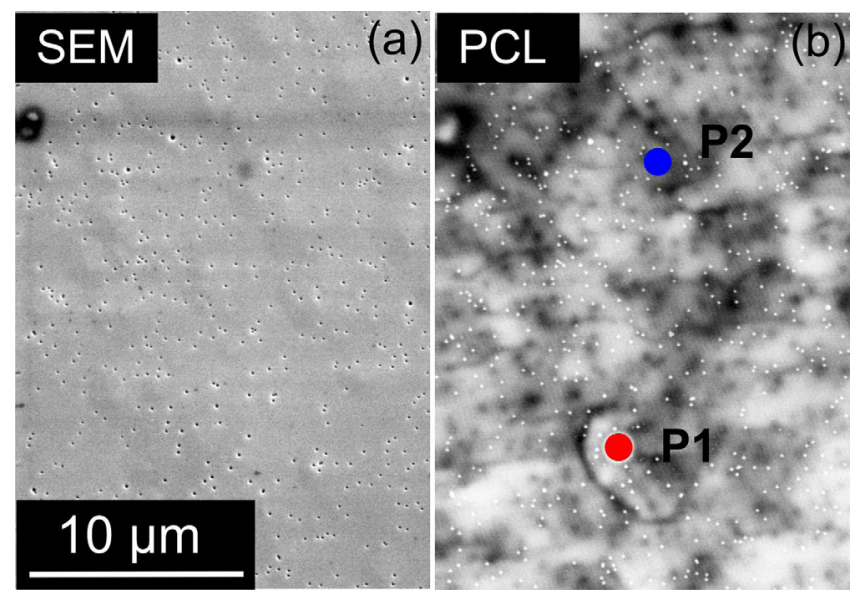

FIG. 3. (a) SEM image and (b) corresponding panchromatic CL image showing two different points with brighter contrast (P1) and with darker contrast (P2).

It is clear that the increase of the MQW intensity vs current measured for the bright spot is faster than the growth of the CL intensity for the dark spot. These results have an obvious correlation with the droop effect and offer a way to spatially resolve it. We would like to point out that these measurements were repeated at different places on the wafer and with varying the magnification; however, the tendency was always similar, i.e., the MQW intensity increases more rapidly for the bright spot compared to the dark spot. Also, any comparison of such data as in Fig. 4 with LED droop data for a fully processed LED should be made with caution, since the LED droop corresponds to the integration of all such local droop studies over the entire LED area.

The experimental data discussed in this work are interesting since they demonstrate (even visually) the relevance of dislocations for droop in violet InGaN/GaN LEDs (and hence in the white LED-lamps). This appears to contradict the mainstream opinion on the cause of droop in the present literature, where defect related processes are largely ignored. $^{1-3}$ The dominating opinion among workers in the field is that the shape of the LI-curve at higher excitation conditions is dominated by intrinsic nonradiative Auger processes. ${ }^{1-3,10-12}$ In our work, we observe that for excitation conditions around the maximum of the LI-curve, the CL intensity is rather homogeneous over the studied area, as expected if the dislocations are shielded from the excited carriers. ${ }^{6}$ This indicates that the dominant recombination here is the intrinsic electron-hole recombination, possibly affected by localization. The only strong nonradiative process apparent in our CL topographs occurs as continuously darkening black spots as the excitation is increased. Such localized recombination events in InGaN LED structures has been studied by the CL technique over the last two decades, and concluded to be related to recombination at dislocation sites. $^{7-9}$ The threading dislocations with a screw component are found to be the most serious nonradiative centers. ${ }^{7,8}$ In a previous work, their connection to droop was not discussed, however.

The relative magnitude of the dislocation contribution to droop for a given LED structure is more difficult to estimate with the present CL technique. Also, as mentioned above,
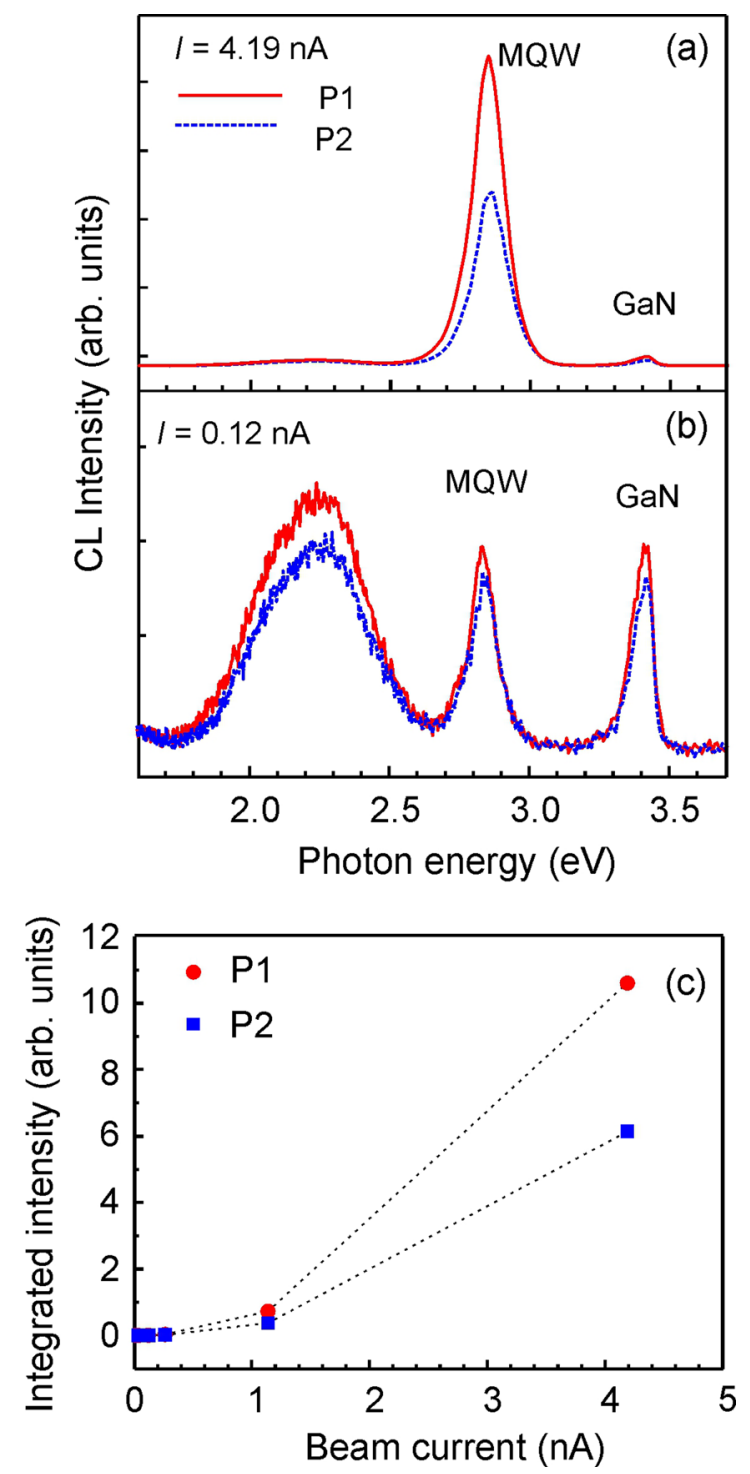

FIG. 4. CL spectra taken at different points P1 (red solid lines) and P2 (blue dashed lines) as marked in Fig. 3(b) corresponding to areas with lower and higher dislocation density, respectively. Spectra are shown for two different beam currents: (a) $4.19 \mathrm{nA}$ and (b) $0.12 \mathrm{nA}$. At high current the MQW emission dominates (a), while at low current (b) the underlying n-layer shows a prominent contribution to the CL emission. (c) Integrated intensity of the CL peak corresponding to the MQW emission, for different currents. The CL intensity shown by red circles is calculated for CL spectra taken in the "bright" point P1, and blue squares correspond to the integrated intensity calculated for spectra taken in the "dark" point P2.

the involvement of intrinsic Auger effects in droop would be observed as a homogeneous lowering of the CL signal across the area studied, as the injection is increased. Such a lowering is difficult to measure accurately in the present CL technique, however.

An attempt to estimate the possible magnitude of the dislocation contribution to droop for different InGaN/GaN LEDs can be made by inspection of published droop data from the literature. In fact there are examples of LEDs with a low droop published in the recent literature, in these cases they were typically grown on a low dislocation density bulk GaN substrate. ${ }^{13,14}$ If we take the cases where the dislocation density is around or below $10^{6} \mathrm{~cm}^{-2}$, we may assume that the dislocation influence on droop can be neglected, and the residual droop in such cases may be understood to be 
dominated by other processes, like Auger effects. As a reference level for typical droop in LEDs grown on foreign substrates we here take the data by Narukawa et al. ${ }^{15}$ The level of droop at the current conditions of $100 \mathrm{~A} / \mathrm{cm}^{2}$ in high brightness white LEDs (see Fig. 5 in Ref. 15) is close to $40 \%$. Here, we define the magnitude of droop as the relative decrease of the light efficiency output with reference to the maximum of the L-I curve. At the same current density, the droop demonstrated in Ref. 13, where low dislocation density bulk GaN substrates were used, is just about 5\% (see Fig. 3 in Ref. 13). Similarly for the case of semi-polar LEDs on bulk GaN substrates, the droop reported for $100 \mathrm{~A} / \mathrm{cm}^{2}$ is just a few percent (see Fig. 3 in Ref. 14). Unfortunately, the design of the LEDs in the three cases discussed was quite different, so a comparison of the values for the droop is not accurate. This still leaves room for the idea that the dislocation related droop process may be responsible for a substantial part of the droop observed in most commercial high brightness LEDs available on the market today. In order to quantify the role of dislocations in the droop process, one needs to produce a series of identical LEDs on substrates with different dislocation density, and measure the droop in this series. Such studies are now in progress.

The conclusion from this study is that the droop in violet or blue InGaN LED emitters shows an obvious contribution from nonradiative dislocation recombination. The relative magnitude of dislocation-related droop in comparison to other contributions ${ }^{1-3}$ has to be established from further studies on dedicated LED structures. On the other hand, the data clearly indicate a way to reduce the droop in these LEDs in the future, via a drastic reduction of the dislocation density common to the mainstream white LED designs, due to the growth on a foreign substrate. This can be done by using bulk substrates, ${ }^{16,17}$ with a sufficiently low dislocation density.

The work at Linköping University was supported by the Swedish Research Council (VR) and the Swedish Energy Agency. The work at Lund University and GLO AB is part of the NanoLund activities.

${ }^{1}$ J. Piprek, Phys. Status Solidi A 207, 2217 (2010).

${ }^{2}$ G. Verzellesi, D. Saguatti, M. Meneghini, F. Bertazzi, M. Goano, G. Meneghesso, and E. Zanoni, J. Appl. Phys. 114, 071101 (2013).

${ }^{3}$ C. Weisbuch, M. Piccardo, L. Martinelli, J. Iveland, J. Peretti, and J. S. Speck, Phys. Status Solidi A 212, 899 (2015).

${ }_{5}^{4}$ B. Monemar and B. E. Sernelius, Appl. Phys. Lett. 91, 181103 (2007).

${ }^{5}$ J. Hader, J. V. Moloney, and S. W. Koch, Appl. Phys. Lett. 96, 221106 (2010).

${ }^{6}$ A. Kaneta, M. Funato, and Y. Kawakami, Phys. Rev. B 78, 125317 (2008).

${ }^{7}$ T. Sugahara, H. Sato, M. Hao, Y. Naoi, S. Kurai, S. Tottori, K. Yamashita, K. Nishino, L. T. Romano, and S. Sakai, Jpn. J. Appl. Phys., Part 2 37, L398 (1998).

${ }^{8}$ T. Hino, S. Tomiya, T. Miyajima, K. Yanashima, S. Hashimoto, and M. Ikeda, Appl. Phys. Lett. 76, 3421 (2000).

${ }^{9}$ C. Cherns, S. J. Henley, and F. A. Ponce, Appl. Phys. Lett. 78, 2691 (2001).

${ }^{10}$ J. Iveland, L. Martinelli, J. Peretti, J. S. Speck, and C. Weisbuch, Phys. Rev. Lett. 110, 177406 (2013).

${ }^{11}$ M. Binder, A. Nirschl, R. Zeisel, T. Hager, H.-J. Lugauer, M. Sabathil, D. Bougeard, J. Wagner, and B. Galler, Appl. Phys. Lett. 103, 071108 (2013).

${ }^{12}$ E. Kioupakis, D. Steiauf, P. Rinke, K. T. Delaney, and C. G. Van de Walle, Phys. Rev. B 92, 035207 (2015).

${ }^{13}$ M. J. Cich, R. I. Aldaz, A. Chakraborty, A. David, M. J. Grundmann, A. Tyagi, M. Zhang, F. M. Steranka, and M. R. Krames, Appl. Phys. Lett. 101, 223509 (2012).

${ }^{14}$ C.-C. Pan, S. Tanaka, F. Wu, Y. Zhao, J. S. Speck, S. Nakamura, S. P. Den Baars, and D. Feezell, Appl. Phys. Express 5, 062103 (2012).

${ }^{15}$ Y. Narukawa, M. Sano, M. Ichikawa, S. Minato, T. Sakamoto, T. Yamada, and T. Mukai, Jpn. J. Appl. Phys., Part 2 46, L963 (2007).

${ }^{16}$ C. Hemmingsson, P. P. Paskov, G. Pozina, M. Heuken, B. Schineller, and B. Monemar, Superlattices Microstruct. 40, 205 (2006).

${ }^{17}$ C. Hemmingsson and G. Pozina, J. Cryst. Growth 366, 61 (2013). 